\title{
Philosophiques
}

\section{Sollen et altérité selon Fichte}

\section{Sur l'intersubjectivité fondamentale, éthique et juridique}

\section{Augustin Dumont}

Volume 43, numéro 2, automne 2016

Dossier. Usages de la réflexivité en philosophie allemande

URI : https://id.erudit.org/iderudit/1038207ar

DOI : https://doi.org/10.7202/1038207ar

Aller au sommaire du numéro

Éditeur(s)

Société de philosophie du Québec

ISSN

0316-2923 (imprimé)

1492-1391 (numérique)

Découvrir la revue

Citer cet article

Dumont, A. (2016). Sollen et altérité selon Fichte : sur l'intersubjectivité fondamentale, éthique et juridique. Philosophiques, 43(2), 273-296.

https://doi.org/10.7202/1038207ar
Résumé de l'article

L'objectif de cet article est d'analyser la mobilisation fichtéenne du concept de Sollen en montrant de quelle manière ce dernier permet à Fichte de lier la dimension catégorique du savoir transcendantal à sa dimension problématique. L'article propose un parallèle entre la mobilisation du Sollen au plan de la fondation du savoir et sa mobilisation au plan de la théorie de l'intersubjectivité. Pour ce faire, l'article superpose trois points de vue : le point de vue fondamental de l'intersubjectivité pure, le point de vue appliqué de l'intersubjectivité juridique et le point de vue appliqué de l'intersubjectivité éthique. 


\title{
Sollen et altérité selon Fichte
}

\section{Sur l'intersubjectivité fondamentale, éthique et juridique}

\author{
AUGUSTIN DUMONT \\ Université de Montréal
}

\begin{abstract}
RÉSUMÉ — L'objectif de cet article est d'analyser la mobilisation fichtéenne du concept de Sollen en montrant de quelle manière ce dernier permet à Fichte de lier la dimension catégorique du savoir transcendantal à sa dimension problématique. L'article propose un parallèle entre la mobilisation du Sollen au plan de la fondation du savoir et sa mobilisation au plan de la théorie de l'intersubjectivité. Pour ce faire, l'article superpose trois points de vue: le point de vue fondamental de l'intersubjectivité pure, le point de vue appliqué de l'intersubjectivité juridique et le point de vue appliqué de l'intersubjectivité éthique.
\end{abstract}

\begin{abstract}
This paper aims at analysing the Fichtean use of the concept of Sollen, by showing how the latter makes possible the way Fichte links the categorical dimension of transcendental knowledge to its problematic dimension. The article draws a parallel between the use of the concept of Sollen regarding the foundation of knowledge and regarding the theory of intersubjectivity. In order to do that, the article juxtaposes three points of view: the fundamental point of view of pure intersubjectivity, the applied point of view of the legal relationship and the applied one of ethical relationship.
\end{abstract}

\section{Introduction}

Dans la recherche fichtéenne des deux dernières décennies, plusieurs commentateurs se sont penchés sur l'étonnante proposition architectonique de la Doctrine de la science, qui avait déjà attiré l'attention des premiers lecteurs de Fichte, sans que ceux-ci n'en tirent toutefois toutes les conséquences ${ }^{1}$. Il s'agit du fameux "Wenn soll... so muss ", dont tout porte à croire qu'il commande la structure du système en son ensemble, le transcendantalisme fichtéen s'inaugurant ainsi dans ce que l'on a pu appeler une «hypothéticité catégorique ${ }^{2} »$. L'objectif de cet article est de proposer une variation sur ce thème essentiel d'un «devoir» (Sollen), dont Fichte semble attendre qu'il préside au geste transcendantal de manière problématique tout en initiant paradoxalement la catégoricité du propos fondationnel.

Le biais que l'on propose est celui de l'intersubjectivité. Fichte en a fait l'un des points nodaux de son activité philosophique depuis ses premières années à Iéna jusqu'aux dernières années de sa production à Berlin. Il est captivant, en effet, de constater que la constitution, par le moi, d'un point

1. Cf. par exemple M. Guéroult, L'évolution et la structure de la Doctrine de la science chez Fichte, rééd. Hildesheim/Zürich/New-York, Olms, I982 (I930), p. I2I.

2. A. Schnell, Réflexion et spéculation. L'idéalisme transcendantal chez Fichte et Schelling, Grenoble, Jérôme Millon, 2009, p. 46. 
de vue «autre», prend la forme d'une sollicitation (Aufforderung) réciproque à agir en vue de la liberté, laquelle est également structurée par le Sollen problématique. Il convient alors, pour prendre la mesure de cette situation, d'interroger dans un même élan le Sollen fondationnel et la doctrine de l'intersubjectivité, déclinée en différents niveaux selon le point de vue systématique privilégié: le point de vue fondamental de la philosophie première, le point de vue appliqué inférieur du droit ou le point de vue appliqué supérieur de l'éthique. Parce que nous sommes convaincus de la profonde continuité de la trajectoire fichtéenne - une appréciation qui n'est certes plus minoritaire aujourd'hui -, nous n'hésiterons pas à faire dialoguer la Doctrine de la science de I 804 (deuxième version), où l'hypothéticité catégorique se voit explicitée de la manière la plus précise, et les textes de l'époque d'Iéna, en particulier la Doctrine de la science Nova Methodo (I796-99), le Fondement du droit naturel selon les principes de la Doctrine de la science (I796-I797) et le Système de l'éthique selon les principes de la Doctrine de la science (I798).

\section{Le «Soll» spéculatif}

La monumentale Doctrine de la science de I $804 / \mathrm{II}$ conjoint, comme on l'a décrit maintes fois, une aléthologie ascendante à une phénoménologie descendante. Prenant son point de départ dans le résidu de factualité qu'il aura toujours dénoncé dans le kantisme (et que manifeste chez Kant la fragmentation des juridictions et des principes), Fichte est ainsi amené à développer tout d'abord une compréhension réaliste de l'être en sa vérité immuable et à opérer pour ce faire une reconduction (Zurückführung) de la multiplicité à l'unité - donnant ainsi en apparence des gages aussi bien à Jacobi qu'au point de vue schellingien de l'identité absolue - , avant de redescendre de l'Un vers le multiple sensible par où le premier se phénoménalise et s'idéalise nécessairement en une "projectio per hiatum ${ }^{3}$ ». Le fil rouge du parcours de I 804 est, comme à l'époque d'Iéna, l'intuition intellectuelle. Désormais baptisée Einsicht - un concept que nous avions proposé de rendre par «introvision compréhensive $e^{4}$ » sur le modèle de l'ancienne intropathie husserlienne -, cette dernière renvoie à la saisie compréhensive, «d'un seul coup (mit Einem Schlage) $)^{5}$, de l'agir du moi se réfléchissant lui-même dans l'unité d'une vision.

La $16^{e}$ conférence constitue le point de basculement d'un mouvement dans l'autre, en un geste qui demeure en réalité proche de la Doctrine de la

3. J. G. Fichte, Die Wissenschaftslehre. Zweiter Vortrag im Jahre 1804, Hamburg, Felix Meiner Verlag, I986, p. I67 (dorénavant: Meiner). Sämmtliche Werke, Bd. X, 218 (dorénavant: $S W)$.

4. Cf. A. Dumont, L'opacité du sensible chez Fichte et Novalis. Théories et pratiques de l'imagination transcendantale à l'épreuve du langage, Grenoble, Jérôme Millon, 20I 2, p. 396.

5. Meiner, p. I5. SW X, 99. 
science Nova Methodo, laquelle s'élevait tout d'abord vers le vouloir pur avant d'en opérer la sensibilisation. À ce niveau d'abstraction, le lecteur ou l'auditeur de Fichte réalise que l'objet de son Einsicht, depuis le début du mouvement ascendant, aura été l'absolu (Absolute) dans son autoconstruction originaire, ou l'être (Sein) comme "Singulum fermé sur lui-même ${ }^{6}$ ", car comprenant en soi toute la réalité. L'être appréhendé ici est l'être dans l'autoconstruction autonome de l'identité absolue de toutes ses différences, mais, ajoute significativement Fichte, il s'atteste seulement «dans la mesure où cette construction doit (soll) être ${ }^{7}$. Qu'une telle construction doive être, voilà ce que l'aléthologie, ou doctrine de la vérité de l'être, accueille en fin de compte comme un fait (Faktum). Plus encore, tandis qu'il cherche la source de ses représentations dans un tel être en soi, lequel semble s'offrir spontanément à lui comme une totalité de sens disponible, le philosophe réaliste (auquel fait droit la première partie de l'ouvrage) doit accorder qu'il a jusqu'ici identifié son propre acte de savoir l'être à l'être lui-même, car il est l'auteur de cette construction de l'être comme Singulum s'autoconstruisant. De la sorte, que l'être se construise tout seul comme unité absolument identique au savoir de l'être, c'est là précisément un fait dont il faut admettre qu'il est posé «à titre problématique ${ }^{8}$ ".

L'aléthologie démontre ainsi qu'il n'y a pas de savoir qui ne présuppose, secrètement ou délibérément, l'identité absolue de l'être, et donc l'identification ultime du savoir à l'être lui-même. Une telle situation factuelle nous assure d'une seule chose: un phénomène quel qu'il soit ne trouvera jamais son fondement ailleurs que dans l'absolu. C'est là une nécessité au sens d'un Müssen catégorique. Toutefois, avance Fichte, "nous qui accomplissons cette construction [de l'être comme s'autoconstruisant] " ${ }^{9}$ ", ou, comme il l'écrira plus loin, "nous, la Doctrine de la science $e^{10}$ ", nous devons opérer une réflexion (Reflexion) sur cette nécessité catégorique. Cette réflexion devra se présenter - Christoph Asmuth y a insisté de façon convaincante ${ }^{11}$ - comme la "répétition (Wiederholung) ${ }^{12}$ » de l'acte par lequel nous accédons à la vérité de l'être clos en soi. Que nous devions réfléchir l'être en son autoconstruction et ainsi le phénoménaliser, c'est-à-dire le faire

6. Meiner, p. I60. $S W X, 212$.

7. Meiner, p. I6I. $S W X, 2$ I4.

8. Meiner, p. I62. $S W X, 215$.

9. Meiner, p. I6I. $S W X, 2$ I4.

10. Meiner, p. 266. SW X, 300. En d'autres termes: nous qui avons rigoureusement effectué chacun des actes exigés jusqu'ici et avons pris conscience que, pour ne pas être l'absolu, nous sommes bel et bien le savoir transitif de l'absolu. Cf. à ce sujet la remarquable étude de M. Marcuzzi, "Nous, la Doctrine de la science", in J. G. Fichte, Doctrine de la science. Exposé de 1813, trad. fr. M. Marcuzzi, Aix-Marseille, Presses Universitaires de Provence, 2014 , p. I 45.

11. Cf. Ch. Asmuth, Begreifen des Unbegreiflichen. Philosophie und Reflexion bei J.G. Fichte, 1800-1806, Stuttgart/Bad-Cannstatt, Frommann-Holzboog, I999, p. I 56.

12. Meiner, p. I 55. SW X, 208. 
être à nouveau dans notre savoir, cela ne relève plus d'aucune nécessité apodictique. Une telle répétition est ainsi créatrice de différence, créatrice d'un nouveau principe, à savoir précisément le Sollen problématique, pendant du Müssen catégorique, et que nous ne voyions pas tant que nous suivions l'unique fil rouge de l'objet du savoir, c'est-à-dire de l'absolu.

La proposition fichtéenne ici à l'étude prend en général la forme suivante: si l'être doit (Soll das Sein...) être posé dans son autoconstruction, alors il doit (so muss...) se donner suivant telle ou telle loi a priori. Dans cette proposition, le "so muss» est pour ainsi dire pleinement acquis au terme du processus aléthologique: s'il nous est possible de comprendre et de donner du sens à ce qui est, ce ne peut être qu'en vertu des lois nécessaires de l'être comme unité absolue (lois entre les mains desquelles le réaliste remet sans hésiter toutes les propriétés de sa conscience, c'est-à-dire de son savoir de l'être), cette dernière résorbant alors, croit-on, la différence entre le sachant et le su. Mais le Soll inaugural (que l'on peut également traduire ainsi: «d $\hat{u} t$-il y avoir une conscience de l'être, alors...») demeure quant à lui non résorbable dans l'unité et problématique. Il ne l'est évidemment pas au sens où l'on se verrait dans l'impossibilité de savoir pourquoi il y a de l'être plutôt que rien. La question métaphysique consacrée par Leibniz ne peut appeler qu'une réponse transcendante, disqualifiée en régime transcendantal, se rapportant à une existence nécessaire. Or l'enjeu est autre. Le problème est plutôt de reconnaître qu'un savoir a priori de la conscience de l'être est ultimement possible et rien de plus. Il n'est certes pas un simple fait (Tatsache) du monde, dont il faudrait partir comme d'un donné irréfléchi. Il s'agit ici d'un Faktum à entendre comme ultime butée du processus génétique de la Wabrheitslehre. Il prend alors la forme d'une exigence (Forderung) du ressort de la liberté humaine, puisqu'il est possible de l'accomplir ou non, et s'offre ainsi comme problématicité (Problematizität) originaire.

Il y a là une liberté contingente qu'il n'y a pas à (et que l'on ne pourrait) fonder en nécessité. Cela supposerait en effet que l'on dispose d'un point de vue supérieur d'où la liberté pourrait être à nouveau déterminée et conditionnée. Or la liberté de l'acte de commencer une série de réflexions et ainsi de phénoménaliser l'être est absolue ou elle n'est pas: c'est bien la liberté, entendue tout à la fois comme possibilité pure et comme exigence absolue dans sa contingence même, qui se détermine, se donne des lois et s'ouvre d'elle-même à la nécessité catégorique. Le Soll factuel est donc ce par où s'inaugure toute phénoménologie, mais il n'ouvre à la catégoricité des lois phénoménologiques qu'en se réalisant et non pas en se constatant, comme y a fort bien insisté Jürgen Stolzenberg: ce qui est exigé (construire un savoir de l'être comme autoconstruction) ne se fonde nulle part ailleurs qu' «à travers l'acte même d'exiger ${ }^{13}$ ", à travers un "Soll extériorisé de

13. J. Stolzenberg, "'Ein neues, bis jetzt noch ganz unbekanntes Prinzip muss aufgestellt werden'. Der Übergang zur Erscheinungslehre in Fichtes Wissenschaftslehre von I804 ", in 
manière performative ${ }^{14}{ }^{\prime}$. Fichte peut alors exprimer le caractère indépassable de cette liaison du Sollen et du Müssen dans l'exposé de I 804:

En ce Soll problématique - ce que je n'ajoute qu'afin d'exciter votre attention -, à entendre comme la fine pointe de notre parcours actuel, tout se trouve ramassé [...]. Il est ainsi clair qu'il faut en rester à cette problématicité instituée dans le Soll comme elle a été instituée. Il n'en reste pas moins qu'il faut parvenir à une catégoricité, sans laquelle notre science dans son commencement et point de départ serait dépourvue de sol et de principe. Mais alors cette catégoricité devrait se retrouver problématiquement dans le Soll en tant que Soll, de telle sorte qu'à présent le principe premier du phénomène (Erscheinung), et comme on peut bien le croire, de l'apparence (Schein), reposerait en ceci que le catégorique absolu, en relation à l'Einsicht, le vrai et le certain, apparaîtrait dès lors comme problématique, c'est-à-dire comme pouvant être ou ne pas être, et pouvant être ainsi ou être autrement ${ }^{15}$.

Le sens de cette méditation fichtéenne est clair à présent. Elle signifie que s’il doit y avoir des lois transcendantales - ce qui est exigé problématiquement en vertu du Soll -, alors ces lois doivent revêtir la forme de la nécessité apodictique - au sens du Müssen cette fois. Toute genèse de l'être est immanquablement une genèse du savoir de l'être, et celle-ci ne peut se présenter comme identique à la première sans reconnaître du même coup qu'elle n'a littéralement nulle raison d'être, sinon tout à la fois la liberté de devoir être et l'exigence de réfléchir cette genèse. Lorsque nous réfléchissons pour elle-même la conscience, qui n'est en fin de compte que le Soll lui-même comme existence (Existenz) ou extériorisation (Äußerung) de l'être par et dans sa libre autoréflexion performative, nous découvrons qu'elle n'est rien d'autre qu'une projection intentionnelle, et que son objet — l'être comme autoconstruction - est "projeté (projicirt) per hiatum ${ }^{16}$ ». Le hiatus entre l'être (ou l'Un) et sa conscience phénoménale (l'apparence de l'Un), consécutif à la projection, nous empêche de parler de l'absolu comme peuvent le faire Spinoza ou Schelling, selon Fichte, c'est-à-dire sans tenir compte de la projection performative, laquelle opère pourtant une scission dans l'identité.

Cette projection, ou intentionnalité, est très exactement à l'image de son principe absolu. C'est ainsi que l'on entre dans la phénoménologie: nous ne connaissons l'être fermé en sa clôture sur soi qu'à travers l'acte de le savoir, c'est-à-dire l'acte par lequel nous le manifestons comme tel en le projetant et ainsi le nions puisque la projection brise sa clôture sur soi. La conscience n'est alors elle-même que l'apparence phénoménale et négative de l'absolu —à laquelle Fichte conférera résolument le statut de «néant » en

J-Ch. Goddard et A. Schnell (dir.), L'être et le phénomène/Sein und Erscheinung. J.G. Fichte, Die Wissenschaftslehre (1804), Paris, Vrin, 2009, p. 374.

14. Ibid.

15.Meiner, p. I67. SW X, 218-219.

16. Meiner, p. I65. SW X, 217. 
I 805 - et ainsi le Soll est-il à l'image de l'absolu. En d'autres termes, le Soll, entendu aussi bien comme possible pur que comme devoir-être inconditionné, repose sur soi, il est en soi et par soi. Comme l'être dont il est le phénomène originaire, il est autocréation et ne dépend donc pas d'un principe extérieur car, explique Fichte, "s'il avait un fondement extérieur, il ne serait pas un Soll problématique mais un $M u \beta$ catégorique ${ }^{17}$ ». Le devoir-être comme exigence absolue de liberté — ou, comme l'on voudra, la liberté comme devoir-être absolu dans sa contingence même - est l'image de l'être, il en a donc la pleine autonomie - une autonomie problématique - car il n'est conditionné par rien; tout à l'inverse, il conditionne lui-même les lois de la réflexion. L'exigence pratique au fondement de toute la Doctrine de la science, explique Fichte, est "une création à partir de rien (Schöpfung aus Nichts) ${ }^{18}$ ", et ainsi se constitue l'expérience sensible toute entière dans le regard du philosophe.

Par la reconstruction réflexive du savoir absolu de l'être ou de l'être comme identique à son savoir absolu (point de vue réaliste), nous le phénoménalisons et lui offrons la forme sensible du Soll, qui n'est rien d'autre que l'idéalisation de l'aléthologie par la phénoménologie (point de vue idéaliste). À cette fin, il aura fallu réfléchir le Soll pour lui-même et en faire un Soll als Soll: dans cette répétition réfléchissante s'indique le savoir en tant que savoir, le phénomène en tant que phénomène. Ainsi Fichte peut-il s'acheminer vers la caractérisation de sa pensée comme un «idéal-réalisme » ou un «réal-idéalisme».

\section{Sur l'intersubjectivité fondamentale}

À condition qu'elle soit continuellement accompagnée d'une Einsicht sans quoi elle demeure lettre morte - la structure propositionnelle du "Wenn soll... so muss» s'avère, on le voit, singulièrement efficace pour caractériser le sens même du transcendantalisme fichtéen comme problématicité catégorique. Lorsqu'il note à son propos, en I804, qu'elle a toujours joué un rôle fondamental, quoique discret, dans son œuvre passée ${ }^{19}$, notre philosophe n'abuse pas ses lecteurs, pas plus qu'il ne s'abuse lui-même. Rappelons par exemple ces lignes du $\$$ I de l'Assise fondamentale de la Doctrine de la science publiée dix ans plus tôt: "Si la proposition $\mathrm{A}=\mathrm{A}[\ldots]$ doit être certaine, alors la proposition: Je suis, doit également être certaine (Soll der Satz... so muß auch der $S a t z . ..)^{20}$ ». De la même manière, l'important $\mathbb{I} 3$ de

17. Meiner, p. I68. SW X, 2 I9.

18. Meiner, p. I68. $S W X, 219$.

19. Cf. Meiner, p. I69. SW X, 220.

20. J. G. Fichte, Grundlage der gesamten Wissenschaftslehre (1794), Hamburg, Felix Meiner Verlag, I997, p. I 5. SW I, 95. Nous soulignons. La profonde cohésion de ces différents points de vue, tout au long de 20 années de production philosophique, sur une même fondation du savoir dans et par l'exigence pratique a été autre autres fort bien mise en évidence par Andreas Schmidt dans: Der Grund des Wissens. Fichtes Wissenschaftslebre in den Versionen von 1794-95, 1804/II und 1812, Paderborn, Schöningh, 2004. 
la Nova Methodo ne peut décrire le vouloir pur comme réunion d'une liberté illimitée et d'une limite pure sans recourir au même stratagème. De quoi s'agit-il ? Le vouloir pur n'est rien d'autre que le devoir (Sollen), c'est-à-dire l'impératif catégorique entendu comme fondement "de la conscience en général ${ }^{21}$ " (là où Kant l'utilise seulement pour expliquer le devoir moral). Il est l'identité absolue du réfléchissant (du vouloir infini) et du réfléchi (du vouloir en tant qu'il se détermine objectivement et se finitise). En clair: pour que la liberté d'agir soit, elle doit se normer, et par ce processus la volonté est tout à la fois déterminée et élevée au savoir de soi, car il faut connaître le concept de fin que l'on assigne à son agir pour pouvoir agir librement; inversement, l'agir ne peut se normer s'il n'est pas déjà, et précisément, libre de le faire, et transcende par avance toute limite objective. Les deux pôles de la contradiction se déterminent réciproquement dans le vouloir pur, c'està-dire dans l'identité pure de l'illimité et de la limite. Cette identité, stricto sensu impensable, se brise immanquablement dans la performance même de notre réflexion sur elle (le vouloir pur est limité et finitisé dans le savoir que l'on peut en avoir), et c'est pourquoi elle est repoussée par la conscience à l'horizon de son activité, comme tâche à réaliser. Ainsi s'explique l'effort (Streben) caractéristique du désir (Begierde). Tout comme pour l'être fermé en soi de I804, rien dans le vouloir pur ne justifie sa libre reprise et sa position par la conscience du philosophe. C'est pourquoi, quant à l'exigence de poser une fois encore cette identité, explique à nouveau Fichte de la façon qu'on a caractérisée, elle "doit $(m u ß)$ nécessairement être admise si la conscience doit être expliquée (wenn [das] Bewusstsein erklärt werden soll $)^{22} »$.

On le voit, en réfléchissant jusqu'au bout les conditions de possibilité de l'acte de penser l'activité du moi, la Nova Methodo ne remonte donc pas non plus au-delà de l'exigence pratique de faire être une conscience libre dans sa volonté s'il doit y en avoir une. Cette possibilité contingente ne saurait être dépassée, étant au contraire le fondement ultime et problématique des conditions de possibilité de la conscience de soi déduites catégoriquement chemin faisant. Ainsi, le sentiment, l'intuition, l'espace, le corps propre, le temps - pour ne citer ici que quelques-uns de ces moments déductifs - sont nécessaires à l'acte d'autoposition et ont donc une validité apodictique si une conscience doit être possible, ce que l'on ne peut fonder en raison, la raison se voyant au contraire elle-même fondée dans le Soll contingent. La conscience naturelle se définit ainsi en dernière instance par ce «doit» lui-même, puisque tout ce que le philosophe transcendantal rencontre dans sa réflexion sur la conscience commune lui est in fine attribué.

21. J. G. Fichte, Die Wissenschaftslehre Nova Methodo, in Gesamtausgabe der Bayerischen Akademie der Wissenschaften (dorénavant: GA), IV, 3, 440.

22. GA, IV, 3, 439. 
Dans ce contexte, il est remarquable que le $\mathbb{S}$ I 3 de la Nova Methodo ne soumette le vouloir empirique - par définition aux prises avec les objets

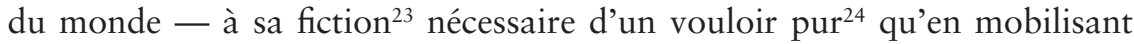
déjà l'altérité, bien qu'elle soit entièrement abstraite à ce stade puisqu'elle n'a pas encore été déduite à proprement parler. Cette altérité pure constitue le déterminable à partir duquel le vouloir opère un choix afin de se déterminer. Dans la mesure où elle est strictement intelligible, il s'agit du "règne des êtres raisonnables hors de moi (Reich vernünftiger Wesen ausser mir) ${ }^{25}$ ". En se déterminant à l'activité pure parce qu'il doit s'arracher à l'inertie (Trägheit) ou au repos (Ruhe), en se donnant le concept qui va normer son agir, le vouloir se singularise. Plus précisément encore: il s'individualise. Dans la mesure où rien de sensible n'est acceptable à ce niveau d'abstraction, puisqu'au contraire l'expérience sensible se fonde sur le vouloir pur, le déterminable d'où le vouloir s'arrache est «un intelligible ${ }^{26}{ }_{\text {}}$ — c'est-à-dire un pur pouvoir d'être un moi en général. Quant au déterminé, c'est l'individu: «je ne peux me trouver à aucune autre condition que celle de me trouver comme individu au milieu de plusieurs êtres spirituels ${ }^{27} »$.

Cela ne suffit évidemment pas à caractériser l'intersubjectivité. Le moi doit repousser au-devant de soi l'accomplissement d'une liberté pleinement identique à elle-même (celle du vouloir pur) dans l'instant même où sa liberté est sensiblement liée (gebunden), donc engagée et divisée dans le vouloir empirique. L'un ne va pas sans l'autre. L'acte même de penser le vouloir pur, en effet, le lie dans le sensible. Dans cette auto-affection de la pensée naît le sentiment du devoir. Le Sollen n'est donc pas un simple concept ratiocinant: il est senti chaque fois que le moi réfléchit son activité. C'est très exactement comme tâche éprouvée, comme devoir senti de s'autodéterminer librement toujours et encore, que le moi s'apparaît à lui-même. Si le règne des êtres raisonnables permet au moi de prendre conscience de lui-même par différenciation d'avec une sphère strictement intelligible, au $\mathbb{\$} I_{3}$, une telle sphère devient ipso facto sensible par la limitation de l'activité. Les $\$$ I4-I9 s'en tiendront à cette seule sensibilisation et inscriront sans trop de diffi-

23. Que le transcendantalisme doive lui-même se penser comme fiction transcendantale est au cœur de nos préoccupations. Cf. A. Dumont, L'opacité du sensible chez Fichte et Novalis, op. cit.

24. Il ne s'agit donc pas de refuser à la liberté son inscription dans le monde, bien au contraire, il s'agit seulement d'éviter que le vouloir se comprenne d'entrée de jeu ou uniquement à partir d'un être prédonné, dogmatiquement posé à l'extérieur, et dont l'existence factuelle oriente le premier de manière déterministe. Le conséquentialisme en serait le premier surgeon en éthique, comme le réalisme en épistémologie. Si le vouloir pur n'existe en réalité que dans sa sensibilisation (on ne peut le penser sans le sensibiliser), insiste Fichte, il est cependant impossible de penser le vouloir empirique sans le vouloir pur en raison des lois de la réflexion transcendantale, et ainsi le second est-il le «modèle» (Vorbild) du premier.

25. GA, IV, 3, 445 .

26. $G A, I V, 3,445$.

27. GA, IV, 3, 445 . 
cultés les éléments constitutifs du vouloir pur dans le vouloir empirique, mais pour cela il aura d'abord fallu admettre une altérité pure, à entendre comme condition de possibilité ultime de la conscience de soi, c'est-à-dire finalement de la pensée du Sollen. Ainsi, s'il doit (soll) y avoir une pensée du Sollen, c'est-à-dire une conscience, alors il est nécessaire que le déterminable de l'activité pure soit la sphère purement intelligible des êtres raisonnables. Toutefois,

les êtres raisonnables hors de moi sont uniquement pensés pour expliquer le divers. Je ne perçois pas la raison et la libre volonté des autres hors de moi, je ne fais que [les] conclure à partir d'un phénomène dans le monde sensible ${ }^{28}$.

Si une conscience doit être possible (proposition problématique), alors un monde purement intelligible d'êtres raisonnables et libres doit être nécessairement (proposition catégorique), bien qu'il ne puisse être en aucun cas perçu. Toutefois, dès qu'il y a réflexion sur ce monde intelligible, un monde sensible est perçu. L'impératif catégorique kantien ne va pas jusque-là, comme le remarque Fichte. En effet,

je dois (soll) agir de telle sorte que ma façon d'agir puisse devenir loi pour tout être raisonnable; mais alors je dois tout de même avoir déjà admis des êtres raisonnables hors de moi, car sinon comment puis-je vouloir rapporter cette loi à ceux-ci ?

L'impératif catégorique revu et corrigé par Fichte doit concilier sa catégoricité avec la problématicité inaugurale: s'il doit y avoir une conscience en général, celle-ci doit nécessairement spécifier et déterminer son activité à partir du pur pouvoir intelligible d'être un moi, lequel devient, dans l'élan même de cette individuation, pur pouvoir d'être un "autre ", c'est-à-dire une conscience individuelle. Qu'il doive y avoir une conscience libre est bien un impératif à portée universelle, mais son contenu catégorique réside seulement dans la nécessité d'un arrachement à la sphère pure des semblables et en l'individuation de la forme de l'autonomie qui lui est attachée. Car quant à la réalisation pratique d'un tel «il doit y avoir», elle est précisément attendue d'une liberté à jamais problématique — c'est-à-dire qu'elle est à la discrétion du sujet qui s'engage pour elle - et bien entendu indémontrable. À cette liberté on ne se rapporte qu'à travers le médium de la croyance en ce que l'on sent (le devoir), en tant qu'un tel sentir, loin d'indiquer seul ce qu'il faudrait faire, est immédiatement réfléchi et ne va donc jamais sans une connaissance toujours en train de se (re)normer et donc de se (re)définir.

Le moi s'arrache ainsi à l'universel pouvoir de vouloir, dont il devient une partie individualisée. Le travail de l'imagination transcendantale consiste à sensibiliser le vouloir pur de telle sorte que tous les éléments déduits pour parvenir jusqu'à ce point suprême ne soient plus seulement des 
conditions de possibilité de l'acte pur de se penser soi-même mais bien des conditions de possibilité de l'acte de penser le monde et de se penser soimême au milieu du monde physique et des autres comme êtres charnels. C'est pourquoi la seconde partie de la Nova Methodo s'attache à redescendre de la condition ultime au conditionné afin de prouver que le vouloir peut bien se diriger simultanément vers quelque chose d'extérieur. Il le peut s'il est retenu par une limitation originaire que le moi doit librement imposer à son activité en réfléchissant sur le vouloir pur. Ainsi le concept de fin pur doit-il devenir concept objectif, et ainsi l'attention pourra-t-elle se focaliser sur l'affection de l'organe externe (le corps matériel), que le moi doit pouvoir répéter et reproduire en lui-même pour qu'elle apparaisse comme vouloir délibérément limité. Le moi est certes libre de s'imposer ou de ne pas s'imposer la limite en question, mais s'il doit être une conscience, alors le pur concept de fin doit devenir un concept objectif, c'est-à-dire la reproduction sensible d'une limite apportée à l'agir. Fichte note de manière prévisible: "mon être (Wesen) est la tâche de faire (machen) [ce concept] sitôt que je réfléchis, mais cela je dois le faire (muß ich thun) si je dois avoir conscience (wenn ich Bewusstsein haben soll) ${ }^{29}$ ".

Reste à savoir comment l'on peut conférer à son activité une limitation en lui donnant un concept objectif. À cette fin, la question de l'intersubjectivité revient dans la seconde partie de la Nova Methodo, au $\$$ I6. Fichte introduit à cet endroit le concept essentiel de sollicitation, de sommation ou encore d'appel (Aufforderung). Il désigne justement l'incapacité pour le moi à se donner seul le concept en question: si tous les éléments précédemment déduits (l'espace, le corps propre, la faculté des concepts, la temporalité, etc.) permettent au moi de se déterminer, c'est-à-dire de conférer une finalité à son activité, il est bien incapable de la déterminer objectivement tant que l'autre ne fait pas irruption. Seule l'altérité peut lui communiquer le concept de ce qu'il doit produire: il s'agit, écrit Fichte, d'une "sollicitation à l'activité libre; celle-ci provient d'un autre être raisonnable semblable à moi, et est jugée de la même manière par lui ${ }^{30}$ ». Il n'y a pas de rencontre avec l'altérité qui ne soit pour Fichte une déstabilisation radicale, tant autrui, loin de s'ajouter simplement à la liste des conditions de possibilité de l'agir libre, incarne l'agir libre du fait même qu'il somme le moi à agir librement à son tour. L'appel ou la sollicitation nécessairement exercée par l'autre, quelle que soit son intention empirique, dès lors qu'il y a une rencontre, apparaît très précisément comme la limite recherchée par le moi pour sa propre activité infinie, à même la perception d'un vouloir infini chez l'autre. Il y a en soi un «manque (Mangel)» ${ }^{31}$, explique Fichte, un manque de l'autre. La liberté ne peut se déterminer si nul ne vient la solliciter, c'est-à-dire aussi bien la

29. GA, IV, 3, 464. Nous soulignons.

30. GA, IV, 3,468 .

31. GA, IV, 3, 469 . 
limiter, non sans se signaler comme volonté en surplus, c'est-à-dire comme pur pouvoir de dépasser la limite, et c'est là la tâche d'un non-moi un peu particulier: le toi.

À nos yeux, la philosophie appliquée intensifie la «dramatique» de la relation intersubjective dont on vient de déployer les grands axes sur un plan fondationnel. À la fin de la Nova Methodo, Fichte propose une Déduction des subdivisions de la Doctrine de la science, contemporaine de sa première philosophie appliquée. La Doctrine de la science doit d'abord offrir une déduction des conditions de possibilité a priori de l'acte de se penser soimême in abstracto. C'est le point de vue de la philosophie première, laquelle livre les principes fondamentaux de l'activité en général. À partir des lois générales ainsi obtenues, le philosophe peut dégager (I) une philosophie théorique ou doctrine de la nature, qui enseigne comment le monde est: elle s'intéresse d'une part au mécanisme (la physique), d'autre part à l'organisme (la biologie); et (2) une philosophie pratique, qui enseigne comment le monde doit être du point de vue de l'être raisonnable en général: l'éthique. On trouve enfin deux points de vue intermédiaires, que Fichte nomme "philosophies des postulats». Il s'agit respectivement de la doctrine du droit et de la doctrine de la religion. Toutes deux se situent entre le point de vue théorique et le point de vue pratique. La philosophie du droit s'enracine dans le point de vue théorique mais elle postule la liberté et adresse ainsi une demande à la philosophie pratique. De son côté, la doctrine de la religion part du point de vue pratique, c'est-à-dire de la liberté (de la moralité comme libre autolimitation), et adresse une demande à la philosophie théorique (en espérant l'accord de la liberté morale et de la nature). Reste le point de vue esthétique, c'est-à-dire le génie du "comme si », lequel permet seul, aux yeux de Fichte, de passer d'un point de vue à l'autre, dans le système, c'està-dire de s'élever du point de vue commun au point de vue transcendantal et de distribuer les différents points de vue appliqués. Le Fondement du droit naturel et le Système de l'éthique n'ont donc pas le même statut et, par suite, leurs points de vue sur l'intersubjectivité ne se recouvrent pas tout à fait. Dans les deux cas, cependant, la théorie de la sollicitation s'y voit redéployée ainsi que le Soll problématique.

\section{Sur l'intersubjectivité juridique}

Fichte, on s'en doute, ne parle de droit naturel qu'en référence à la tradition moderne du jusnaturalisme ${ }^{32}$, car, dans le Fondement, une fiction chasse l'autre: s'il n'y a plus guère de place pour la fiction de l'état de nature, l'application systématique du concept de droit ou doctrine du droit propre-

32. «Il n'y a par conséquent, au sens où l'on a pris souvent ce mot, absolument aucun droit naturel, c'est-à-dire qu'il n'y a pas de rapport juridique possible entre humains en dehors d'une communauté et sous des lois positives». GA, I, 3, 43 I-432. Tout à l'inverse, "l'État luimême devient l'état de nature de l'humain ". GA, I, 3, 432 . 
ment dite (troisième section), faisant suite à la déduction du concept de droit pur, présentera ainsi la fiction des "droits originaires (Urrechte)" comme fondement de toute doctrine juridique digne de ce nom: «Un droit originaire est ainsi une fiction (Fiktion) pure et simple, mais elle doit, pour l'exercice de la science, nécessairement être élaborée ${ }^{33}$.» Une telle fiction est celle d'un droit absolu, non négociable, propre à tout individu du fait même de son appartenance à la sphère des êtres raisonnables - à entendre non pas au sens d'une communauté particulière mais justement abstraction faite de celle-ci, donc en regard du pur pouvoir d'être un moi doué de liberté. Ce droit est, pour une volonté, celui d'être absolument cause dans le monde sensible (entendu comme sphère infinie des fins du moi) et de ne jamais être un simple effet déterminé de l'extérieur. Mais cela ne vaut pour le moi que parce que cela se présente tout d'abord chez l'autre qui le sollicite et, par suite, le droit positif ne pourra traduire ce droit originaire qu'en fixant ou en quantifiant concrètement la co-limitation des libertés par autrui dans le sens d'une égalité du droit à être cause. L'État sera alors la figure du tiers à laquelle l'on remet le pouvoir de décider de la bonne applicabilité de ce droit originaire et de contraindre celui qui viole délibérément un tel partage déterminé du droit d'être cause. Il convient d'accepter cette fiction si une science du droit doit être possible, et par suite de juger à son aune la légitimité de tout type d'action juridique. Une telle fiction ne renvoie plus à aucun «fait de nature» - la nature s'étant contentée de poser côte à côte les êtres raisonnables et libres -, mais exprime déjà, et bien au contraire, par la forme juridique même, l'exigence d'une liberté collective réelle, indissociable du droit politique et donc de l'État. La force contraignante du droit ainsi institué et garanti par l'État lui confère en apparence une nécessité semblable à celle du monde naturel étudié par la physique, bien qu'elle soit le fruit d'une libre institution, à la fois soucieuse d'adapter ou de réécrire constamment l'équilibrage des volontés se déterminant réciproquement et attachée à lui donner le statut d'un mécanisme naturel parfaitement réglé. On se rapporte ainsi au droit, et plus fondamentalement à l'État, comme s'il s'agissait d'une nature, certes parfois capricieuse ou imprévisible mais en réalité toujours réductible à un corps de lois, alors même que le monde juridique est le fruit d'un agir libre et auto-instituant, postulant la liberté absolue de tous.

L'intersubjectivité fait son apparition dès la première section de la première partie du Fondement, qui s'attache à une déduction du concept de droit - entendu comme condition de la conscience de soi - , et se voit élaborée dans la seconde section, qui s'occupe de déduire l'applicabilité du concept de droit. Il ne s'agit certes pas pour Fichte de combler par la philosophie du droit une lacune de la philosophie première, laquelle prétend bien offrir l'ensemble des conditions de possibilité de la pure conscience de soi. La déduction proposée dans le système juridique de Fichte a un statut diffé- 
rent. Comme l'a bien mis en évidence Ives Radrizzani, le concept de droit n'est pas condition de la conscience de soi au sens où «il ajouterait une nouvelle condition à la liste établie dans la science supérieure, mais où il trouve le moyen de résoudre des contradictions qui pourraient surgir entre des conditions appartenant à cette liste ${ }^{34}$ ». Le droit apporte ainsi « un modèle de règlement des conflits entre des conditions potentiellement contradictoires $^{35}$ ». En effet, la nécessité — acquise au plan transcendantal, comme on l'a montré plus haut — de poser la sollicitation d'autrui si une conscience de soi doit être possible, participe certes de la fondation du savoir pur, dans la Doctrine de la science, mais ne nous informe nullement de la viabilité empirique de la coexistence des êtres raisonnables se sollicitant réciproquement, ce dont le point de vue appliqué de la science du droit peut en revanche se charger.

Si l'autre se phénoménalise comme vouloir infini incarné, c'est-à-dire identique à la limite de son corps propre, et si c'est bien là ce qui limite en retour l'agir infini du moi, si l'autre est lui-même la coalescence de la limite et de l'illimité, le vouloir pur posé par le moi à l'horizon de son agir prend d'entrée de jeu la forme de l'altérité qui le sollicite à l'activité. Toutefois, parce qu'il incarne la même chose pour son autre, ni ce dernier ni le moi ne sont l'identité absolue du vouloir infini et de la limite: ils ne peuvent que se prêter réciproquement le concept de la liberté en bornant leur agir par l'autre, grâce auquel la liberté d'un tel agir s'apparaît à elle-même. Cette situation originale appelle nécessairement une organisation juridique si la conscience doit pouvoir faire l'épreuve concrète de l'altérité — c'est là ce que la philosophie première laissait indéterminé. Si la liberté du moi tout à la fois s'arrête et commence avec la liberté de l'autre, comment faut-il moduler cette limitation de la liberté propre au moi ou, en des termes plus immédiatement fichtéens, comment peut-on quantifier l'agir à supprimer en soi pour laisser advenir la liberté de l'autre, et vice versa? La science du droit postule ainsi la possibilité d'une coexistence réelle et achevée des libertés, qu'il lui faut s'acharner à faire être en proposant ses modulations de l'exercice commun de la liberté.

Il faut le souligner, la «proposition du droit ${ }^{36}$ », à savoir qu'il n'y a de relation juridique que dans l'autolimitation d'une liberté par le concept de la possibilité de la liberté de l'autre, et inversement, ne peut se dire qu'en référence au possible et demeure à ce titre problématique: il y a relation juridique si et seulement si chacun limite sa liberté par la simple possibilité de la liberté de l'autre — que le moi reconnaît par là-même comme son sem-

34. I. Radrizzani, «Droit et nature. Le droit naturel chez Fichte", in Recherches sur la philosophie et le langage, dossier sous la direction de J.-M. Lardic, "Fichte: idéalisme, politique et histoire ", Grenoble, 2003 , n²2, p. I 44.

35. Ibid.

36. GA, I, 3, 358 . 
blable ${ }^{37}$ - ce qui est certes exigé mais n'est pas fondé apodictiquement. Aussi ultimement problématique que les droits originaires qu'elle fonde, la proposition primitive du droit n'est pas seulement une fiction régulatrice (au sens où ce qu'elle énonce n'est jamais parfaitement traduit dans l'application et fait donc l'objet d'une visée téléologique), elle est aussi bien une fiction constitutive, qu'il a fallu se donner à soi-même pour qu'il y ait une conscience de soi viable empiriquement. Puisque le moi ne peut se décider à agir en vue d'un concept de fin — c'est-à-dire de quelque chose qui, n'étant d'aucune manière, doit être — que dans la mesure où l'autre, incarnant sensiblement un tel agir téléologique, le sollicite à se former le concept de sa liberté, nul système juridique ne pourra outrepasser son ancrage dans l'acte contingent de suspendre la possibilité de son agir à la possibilité de l'agir de l'autre. Mais il faudra bien que la conclusion soit catégorique: $s i$ il y a cette reconnaissance de la possibilité de l'autre, alors le droit est possible en dernière instance. Fichte indique très tôt, dans le traité, qu'il faudra veiller à passer toujours du problématique au catégorique, car le concept de droit ne fait qu'exprimer de son point de vue appliqué le problème de la relation intersubjective comme telle:

Mais l'individu C ne peut avoir agi sur moi de la manière décrite sans m'avoir reconnu (anerkannt) au moins problématiquement (problematisch); et je ne peux le poser comme agissant ainsi sans poser cela (qu'il me reconnaît au moins problématiquement ${ }^{38}$.

Et le philosophe d'ajouter aussitôt: "Tout ce qui est problématique devient catégorique quand la condition s'y ajoute ${ }^{39}$ ». Si le moi, présupposant d'abord la liberté de l'autre à titre hypothétique, le reconnaît, c'est-à-dire le «traite $(\text { behandelt })^{40}$ » comme un être raisonnable doué de liberté et limite ainsi sa liberté propre, alors l'autre est catégoriquement obligé de reconnaître le moi à son tour - et vice versa. Ce Sollen est clairement l'imitation, à un niveau inférieur, du Sollen spéculatif inaugural, auquel tout le processus gnoséologique est suspendu chez Fichte. En sollicitant l'autre, le moi limite la sphère de son agir possible, ménageant ainsi une place pour la réponse imprévisible de l'autre - et inversement. L'obligation de reconnaître le moi est, pour l'autre ainsi sollicité, catégorique, mais cela ne signifie nullement que la reconnaissance en elle-même le soit.

Il est à ce titre remarquable de voir Fichte méditer de façon soutenue la liaison du problématique et du catégorique dans la rencontre avec autrui, en montrant comment et sur base de quoi la catégoricité est conclue. Ce n'est là rien de moins que le sens même de la Déduction de l'applicabilité du

37. Au sujet de la reconnaissance, cf. notamment: F. Fischbach, Fichte et Hegel. La reconnaissance, Paris, Presses Universitaires de France, I999.

38. GA, I, 3, 353 .

39. GA, I, 3, 353 .

40. GA, I, 3, 353 . 
concept de droit $(\$ 5-7)$ : la proposition du droit, définissant la relation juridique au sens minimal, n'est applicable que dans la mesure où la reconnaissance d'autrui peut être réelle. Une liberté posée seulement à titre hypothétique chez l'autre n'ouvre à aucun système juridique contraignant. Ce dernier ne doit son pouvoir de contrainte qu'à l'acceptation d'une liberté réelle partagée, que l'on ne peut présupposer déjà acquise pour fonder le droit puisqu'au contraire le droit est suspendu à sa reconnaissance. Comme le note Quentin Landenne,

il ne peut être juridiquement obligatoire d'entrer en relation juridique; la normativité juridique, pour pouvoir être opératoire, présuppose pragmatiquement cette entrée en relation comme un fait donné dans sa manifestation externe, abstraction faite de la disposition d'esprit (Gesinnung) ou de la bonne volonté des individus en relation ${ }^{41}$.

Le système juridique, contraignant par nature, ne peut contraindre que celui qui, reconnaissant de facto la liberté d'autrui, se pose également en être raisonnable. Cette dimension factuelle et pragmatique de la reconnaissance qui constitue à proprement parler la catégoricité de l'obligation juridique, engage une profonde réflexion sur le corps. Il ne s'agit pas de concevoir abstraitement l'autre comme un semblable: "traiter " l'autre comme un être raisonnable signifie lui attribuer un corps propre dans l'élan même où le moi s'en attribue un. Le moi et son autre s'attribuent tous deux librement une sphère d'activité dans le monde, qui puisse leur être à chaque fois propre, et qui est projetée par l'imagination productrice. Cette sphère n'est autre que le corps à titre de partie du monde inaliénable, comme réservoir de possibles en perpétuel développement dans l'espace et le temps. Le moi s'attribue l'intégralité de cette sphère, y compris là où elle est entravée: le corps ne ressaisit sa liberté propre que s'il saisit l'obstacle comme une occasion pour sa liberté de s'exercer potentiellement; il doit donc supprimer intérieurement l'agir qui aurait lieu s'il n'y avait pas l'empêchement, et qui pourrait aussi bien avoir lieu. En effet, la volonté pure du moi, ou organe supérieur, négocie constamment avec l'organe inférieur (le corps comme enveloppe sensible en commerce avec la matière) afin de se réapproprier ses interactions, de les reproduire intérieurement et de s'attribuer aussi bien l'initiative du mouvement. Tout le matériel perceptif fait l'objet d'une réflexion autonome de la volonté, continuellement agissante, même là où il y a entrave puisque cette entrave est pour elle, tandis que l'organe inférieur peut être arrêté purement et simplement par l'obstacle sensible.

Il est patent que la plus rudimentaire perception de l'autre et de son corps articulé (par où le moi ressaisit son propre corps articulé, indéfiniment déterminable et modifiable) n'est pas la perception d'une matière brute: le

41. Q. Landenne, Le perspectivisme transcendantal de Fichte. Les points de vue de la liberté, de la spéculation à l'application, Hildesheim/Zürich/New-Yorg, Olms, 20I3, p. I 20. 
corps de l'autre est d'abord perçu comme motilité habitée par une intentionnalité et traversée par des flux de sens, qui sont autant de déterminations de sa volonté, c'est-à-dire qu'on le voit agir comme s'attribuant identiquement une sphère d'activité propre. Par suite, le moi ne devient pas une "personne » parce qu'il s'attribue nécessairement cette sphère mais bien parce qu'il en perçoit le processus d'attribution chez l'autre: «Ce transfert (Übertragen) de ma pensée nécessaire sur une personne (Person) hors de moi repose également dans le concept de personne ${ }^{42}$.» Parce qu'il est impossible, pour le moi, de ne pas être immédiatement affecté en son organe supérieur - c'està-dire sollicité - par cette matière au statut bien spécifique qu'est le corps propre de l'autre, là même où ce dernier ne modifie pourtant point l'organe inférieur (qu'il peut toutefois limiter dans le cas d'une entrave physique), Fichte décrit l'intentionnalité opérante à même le corps matériel de l'autre non comme de la matière brute, mais au contraire comme de la «matière subtile (subtile) ${ }^{43}$ ", c'est-à-dire modifiable par la seule volonté mais absolument coalescente au corps matériel et uniquement perçue.

La perception n'est toutefois pas tout: il nous faut «traiter» l'autre, on y a déjà insisté, comme un être raisonnable en plus d'être affecté au plan du régime perceptif. Se joue ici une dimension pragmatique essentielle chez Fichte. La liberté de la «réponse» pratique à l'appel ou à la sollicitation de la matière subtile perçue est en jeu, une fois qu'a été admise son existence de facto:

s'il dépend seulement de moi que je veuille ou non m'abandonner à cette influence; davantage même, s'il dépend seulement de moi de vouloir, de manière générale, produire de l'effet en retour, et de choisir comment, la possibilité de cette expression de ma liberté dépend du fait que se produit l'influence de l'autre ${ }^{44}$.

Une fois admise l'influence de l'autre, le moi doit choisir comment répondre, c'est-à-dire agir concrètement dans la sphère intersubjective en faisant de l'autre un être raisonnable qui traverse une épreuve identique à celle du moi. Toute la rationalité, ajoute cependant Fichte, semble alors dépendre du hasard, c'est-à-dire de la bonne volonté d'un autre, le moi autonome, tout comme sa réponse à la demande qui lui est adressée, n'étant que l' "accident (Accidens) ${ }^{45}$ » d'autrui. Cette accidentalité empirique est inéliminable, bien que l'on puisse imaginer l'agir du moi préalable à toute rencontre avec autrui comme un pur pouvoir (Vermögen) d'agir, un réservoir d'actions potentielles qui ne serait toutefois doué d'aucune causalité sur le plan de l'organe supérieur, sa causalité se réduisant à sa simple inscription matérielle

42. GA, I, 3, 374 .

43. GA, I, 3, 37I.

44. GA, I, 3, 375 .

45. GA, I, 3, 375 . 
dans l'espace en l'absence de toute sollicitation. À tout le moins, le moi qui ne serait pas encore une personne - le moi non sollicité - est préparé à recevoir la sollicitation et à répondre. Fichte cherche ici à montrer que, si l'éveil du moi dépend assurément de l'influence d'un autre, cet autre est toujours pour ainsi dire influencé à exercer son influence, même en l'absence - posée à titre fictif — de garantie d'un organe supérieur chez son interlocuteur. Ce niveau d'analyse est celui de l'anthropologie, explique le philosophe, et ce point de vue oriente les pages conclusives de la deuxième section. À la fois fortes, originales et parfois naïves, ces pages visent à démontrer à l'aide de références à la plante, à l'animal, puis au visage et même au regard, qu'un corps humain, produit de la nature organisé mais inachevé, se présente comme règne des fins à jamais non totalisable: «tous les animaux sont achevés, et terminés, l'humain est seulement esquissé et projeté [...] Chaque animal est ce qu'il est; l'humain seul, originairement, n'est absolument rien. Ce qu'il doit être, il lui faut le devenir ${ }^{46}$." Si Fichte ne se départit pas des préjugés de son temps sur l'animal comme immobilité et clôture sur soi $^{47}$, il veut en réalité suggérer sa perfection de manière à tout le moins originale dans le contexte de l'époque. Loin de "manquer» de tout ce qui fait la richesse de l'homme — c'était là pourtant le crédo de la tradition — l'animal est seul une «œuvre ${ }^{48}$ » de la nature parce que pleinement identique à son concept, là où l'humain est complètement délaissé par celle-ci et ne devient intégralement que par la sollicitation.

Empiriquement contingente, la sollicitation d'autrui est transcendantalement nécessaire s'il doit y avoir une conscience de soi, tout comme le passage d'une liberté problématiquement posée hors de soi à sa position catégorique dans l'autre est nécessaire s'il doit y avoir une relation juridique. Là réside la complexité de l'intersubjectivité juridique chez Fichte, car l'être relationnel ne fait que manifester au niveau empirique le Soll fondamental en manifestant à la fois l'impératif qu'une conscience soit — la sienne grâce à celle de l'autre et vice versa - et l'énigme de cette conscience, dont il sait qu'en dernière instance elle est toujours problématiquement possible, et que celui à qui s'adresse sa demande doit accepter d'être modifié par cette dernière pour répondre en première personne comme être de droit. En réalité, autrui ne fait qu'adresser une question à son correspondant en même temps qu'il lui transmet son exigence, et c'est là l'impulsion nécessaire à la mise en branle de sa propre activité. Pour ouvrir le moi à sa liberté propre, la question émanant de l'autre vient aussi bien l'assujettir. Voilà une expression à prendre à la lettre: le moi se constitue pragmatiquement comme réponse à la question qui le convoque, et ainsi seulement devient-il sujet de sa liberté

46. GA, I, 3, 379 .

47. Ce sera un trait caractéristique du romantisme postfichtéen de Novalis que de vouloir donner au «toi » une extension plus large que celle de Fichte.

48. GA, I, 3, 379 . 
- un sujet précisément libre de questionner la liberté de l'autre et la sienne propre et d'en répondre comme il l'entend. Par suite, tout système juridique contraignant sourd du "prêt » de la liberté: la sollicitation à entrer dans une relation de droit avec autrui demeure dépendante de l'imprévisibilité de la réponse qui est retournée et constitue comme telle une interprétation - irréductible à la question initiale et lui apportant un surcroît de sens - de ce que signifie être sujet. Même dans la violence ou la contrainte physique, le bourreau manifeste plus que jamais sa terreur de la réponse qui serait venue de l'autre s'il n'avait cherché à anéantir son libre pouvoir de répondre - en droit incompressible - , il opère pour ainsi dire la confirmation performative de ce que sa liberté est entièrement suspendue à celle de l'autre. La sollicitation comme porte d'entrée dans la relation juridique constitue ainsi le passage de témoin du Soll fondamental par lequel nous avons commencé, plaque tournante de la libre phénoménalisation de l'être. Le moi offre à son interlocuteur une limite sensible, phénoménale, en l'appelant à se poser librement et à agir dans le monde, mais ce qu'il lui prête n'est rien de donné ou d'achevé, c'est une exigence d'autant plus précaire qu'elle échappe à toute prévision de ce qu'autrui pourra offrir en retour comme réponse et comme compréhension du concept de liberté. Le prêt est d'autant plus risqué qu'il présuppose qu'autrui reçoive précisément la liberté comme un prêt et non comme un objet qu'il pourrait posséder. Voilà pourquoi l'éducation joue un rôle fondamental dans la pensée de Fichte. C'est parce que l'autre est pour le moi un problème sans solution autre que celle qui lui reste toujours encore à imaginer qu'il est libre d'initier sa propre réponse, donc d'agir dans un monde juridique. Ce dernier n'en demeure pas moins ultimement frappé du seau de la contingence, comme le signale Fichte en conclusion de la seconde section, juste avant de passer à la doctrine du droit proprement dite :

Aucune raison absolue ne se laisse indiquer pour laquelle chacun devrait (sollte) faire de la formule du droit: restreins ta liberté de telle manière que l'autre, à côté de toi, puisse aussi être libre, la loi de sa volonté et de ses actions. Il se laisse aussi bien comprendre (einsehen) qu'une communauté d'êtres libres en tant que tels ne pourrait subsister si chacun n'est pas soumis à cette loi; et que, par conséquent, qui veut cette communauté devrait (müsse) nécessairement aussi vouloir la loi; qu'elle a donc une validité hypothétique. $S i$ une communauté d'êtres libres en tant que tels doit être possible (möglich seyn soll), alors la loi juridique doit valoir (so muß...gelten $)^{49}$.

\section{Sur l'intersubjectivité éthique}

Il n'y a chez Fichte, en rupture avec la tradition sur ce point, aucune déduction du droit à partir de l'éthique. Le droit, point médian entre ce qui est et 
ce qui doit être, adresse une demande à l'éthique en la priant de l'informer de sa liberté pratique pure, tandis que l'éthique, fine pointe de toutes les applications de la science fondamentale, doit présupposer qu'il y a déjà du droit «au dehors» - autrement dit que les interactions au sein de la société civile sont régulées - pour pouvoir s'ériger en normativité strictement attachée au devoir-être «à l'intérieur» de l'individu. L'éthique n'a plus rapport à la simple régulation des interactions et à la négociation du "permis » et du «défendu» à partir du découpage des limites assignées aux libertés — de telle manière que le droit apparaisse comme une seconde "nature» - mais elle se rapporte à l'exigence infinie de transformer l'intégralité du monde en liberté absolue. Le monde de la liberté ne peut bien entendu apparaître comme seulement "permis » par l'établissement de règles juridiques contraignantes, mais il doit être absolument par l'établissement d'une normativité éthique en raison même de la structuration de la conscience établie génétiquement dans la Doctrine de la science. Le droit est suspendu à la reconnaissance de la liberté, mais il a seulement pour mission de réguler la cohabitation des êtres raisonnables, c'est-à-dire des différentes incarnations de la liberté, qui s'entrechoquent nécessairement.

Le Système de l'éthique met donc l'accent sur l'agir individuel et s'occupe de déduire l'obligation morale, dont il fait une condition nécessaire de la conscience de soi. Son principe fondamental est la capacité de la raison à se donner sa propre fin absolument. S'agissant, avec le traité de I798, d'offrir un savoir génétique de ce principe, le point de départ de la philosophie de l'éthique sera la pensée pure et nécessaire, la "pensée première et $a b s o l u e^{50}$ » - fondée sur l'intuition intellectuelle de l'activité absolue selon laquelle le moi doit intégralement déterminer sa liberté d'après le concept de l'autonomie (Selbstständigkeit):

Le contenu de cette pensée est que l'être libre doit être, car le devoir-être (Sollen) est justement l'expression pour la détermination de la liberté; qu'il doit placer sa liberté sous une loi; que cette loi n'est autre que le concept de l'autonomie absolue (indéterminabilité absolue par quoi que ce soit en dehors de lui); enfin, que cette loi vaut sans exception parce qu'elle contient la détermination originaire de l'être libre ${ }^{51}$.

Plus sommaire que le Droit naturel sur les différents aspects du corps articulé comme véhicule de la liberté, le traité de I798 insiste en revanche sur le caractère profondément pulsionnel de ce corps dont la naturalité est fortement soulignée. Le moi peut ainsi affirmer: "cette nature mienne est une pulsion (Trieb) $)^{52}$ », et même une «pulsion à l'auto-conservation ${ }^{53}$ ». La naturalité n'est toutefois que l'envers de la liberté, la pulsion étant aussi bien

50. GA, I, 5, 69.

51. Ibid., 70.

52. Ibid., , го8.

53. Ibid., I I 8 . 
pulsion à l'autonomie selon le point de vue que l'on sélectionne. En effet, en revenant réflexivement sur l'activité du moi, c'est-à-dire sur l'identité du sujet et de l'objet de la réflexion comme devant être, le moi philosophant peut s'attacher à son aspect objectif comme à son aspect subjectif. Le moi comme objectivité est le vouloir en prise sur un donné et intégralement compréhensible à partir du tout de la nature organisée, dont il s'offre comme une partie, tandis que le côté subjectif de l'activité réside dans la capacité à se savoir réflexivement producteur du concept du vouloir indépendamment des objets à partir desquels celui-ci se comprend objectivement. Ce n'est d'ailleurs que parce que la volonté se sait elle-même, ce n'est que parce qu'elle est réfléchie, qu'elle est à proprement parler pulsion ${ }^{54}$. On ne retracera pas ici tout le parcours qui mène à la caractérisation de la faculté supérieure de désirer au $\mathbb{\$}$ IO. La conclusion du $\$ 9$ le résume bien:

Ma pulsion (Trieb) comme être naturel, ma tendance (Tendenz) comme pur esprit, sont-elles deux pulsions différentes? Non, au point de vue transcendantal, toutes deux sont une seule et même pulsion originaire (Urtrieb), qui constitue mon être: elle est seulement vue de deux côtés différents. En effet, je suis sujet-objet et mon être véritable consiste dans l'identité et l'inséparabilité des deux. Si je m'aperçois (Erblicke ich mich) comme un objet complètement déterminé par les lois de l'intuition sensible et de la pensée discursive, ce qui est en fait mon unique pulsion devient pour moi pulsion de la nature, parce que, sous cet angle de vue (Ansicht) je suis moi-même nature. Si je m'aperçois comme sujet, elle devient pour moi pure pulsion spirituelle; ou bien loi de l'autonomie. C'est seulement sur l'action réciproque de ces deux pulsions, laquelle est en fait seulement l'action réciproque d'une seule et même pulsion avec soi-même que reposent tous les phénomènes du moi [...]. Toutes deux sont en fait une seule; cependant, l'égoïté toute entière repose sur ceci qu'elles apparaissent (erscheinen) distinctes. La séparation de la frontière entre les deux est la réflexion (Reflexion) ${ }^{55}$.

Fichte précise alors que le point de vue de l'objectivité de la pulsion — c'està-dire du réfléchi — est celui de la nature comme faculté inférieure de désirer, tandis que le point de vue subjectif, celui du réfléchissant, exprime la faculté supérieure de désirer, comme désir de la raison pour elle-même:

La pulsion supérieure, comme pulsion du pur spirituel se dirige vers l'autodétermination absolue à l'activité pour l'activité, et ainsi s'oppose de manière conflictuelle à toute jouissance, qui est un pur et simple abandon à la nature.

Cependant, toutes deux constituent seulement un seul et même moi; par conséquent, les deux pulsions doivent être réunies dans le champ de la

54. Cf. à ce sujet J.-Ch. Goddard, "Pulsion et réflexion chez Fichte. Une éthique pulsionnelle ", in J.-Ch. Goddard, La Pulsion, Paris, Vrin, 2006; A. Dumont, «La genèse de l'affectivité: tendance, pulsion et sentiment chez Fichte à l'époque d'Iéna ", in Philosophiques, $20 \mathrm{II}$, vol. $38, \mathrm{n}^{\circ} 2$.

55. GA, I, 5, I25-I 26. 
conscience. Il sera démontré que, dans cette réunion, la pureté de l'activité (la non-détermination par un objet) doit être abandonnée par la pulsion supérieure, la jouissance comme fin doit être abandonnée par la pulsion inférieure; de telle manière que se trouve l'activité objective comme résultat de la réunion, dont la fin dernière est l'absolue liberté, l'absolue indépendance de toute nature: c'est là une fin infinie, jamais atteignable; partant, notre tâche ne peut être que celle d'indiquer comment il faut agir pour s'approcher de cette fin dernière ${ }^{56}$.

Et Fichte de s'atteler d'abord à une caractérisation plus précise de la faculté de désirer, avant de se pencher sur les conditions formelles de la moralité puis ses conditions matérielles, les unes et les autres étant contenues dans le concept d'éthique. Ne considérer que la faculté supérieure de désirer serait se condamner au formalisme vide de l'éthique kantienne: une éthique "réelle ${ }^{57}$ ", selon Fichte, conjoint le matériel et le formel, c'est-à-dire les deux faces d'une même pulsion, pulsion de la nature et pulsion de la liberté. C'est pourquoi Fichte définira, au $\$ I2, la "pulsion morale (der sittliche Trieb) » comme une "pulsion mêlée (gemischter Trieb) ${ }^{58}$ ": la vie sensible et les objets matériels sont immanquablement les partenaires de la volonté, mais sa fin dernière est sa propre et absolue indépendance. Il ne s'agit pas de fonder l'éthique sur l'obéissance à une pulsion, fût-ce la pulsion morale, mais de comprendre qu'il n'y a rien en éthique qui ne soit pulsionnel. S'il faut obéir à l'impératif catégorique, c'est-à-dire à un concept de fin pur, qui est en fin de compte la loi de la liberté elle-même ou bien la liberté comme loi, seule la pulsion morale pousse, littéralement (treibt), à ce que l'on s'en forme le concept et rend possible l'intégration de ce dernier dans la conscience. Ainsi se forme la conscience morale (Gewissen) et son intimation à obéir au devoir avec conviction (Überzeugung), l'effort ou l'aspiration (Streben) à accomplir la liberté s'apparaissant à la fois comme produit de la nature et comme poussée libre selon le point de vue que l'on privilégie.

On ne s'étonnera pas de ne pas retrouver dans le Système de l'éthique la richesse des pages que la Nova Methodo ou le Droit naturel consacrent à l'intersubjectivité. D'une façon bien protestante, l'éthique fichtéenne trouve d'abord son lieu dans l'intériorité de la conscience individuelle, dans le «face-à-face» entre la conscience et la loi qu'elle se donne. Si le système des devoirs n'a de sens que pour un individu socialisé, donc soucieux d'agir pour la liberté au milieu des autres et avec eux — nous y reviendrons - , le problème de la cohabitation avec autrui est d'abord l'affaire du droit. De plus, il n'est pas anodin que l'éclipse de la figure de l'autre dans les deux premières parties s'accompagne d'une suspension apparente de la problématicité au profit d'une catégoricité pure et simple. Agir selon l'impératif

56. GA, I, 5, I26.

57. GA, I, 5, I 26.

58. GA, I, 5, I43. 
catégorique, en effet, ce n'est pas agir pour telle ou telle raison contingente: cet agir "doit (soll) être absolument parce qu'il doit (soll) être. Ce devoir (Sollen) est par conséquent un devoir catégorique absolu ${ }^{59}$ ». Une telle règle de l'autonomie, ajoute significativement Fichte, est "une loi valable sans exception, étant donné, en effet, que sa validité n'est subordonnée à aucune condition possible ${ }^{60} »$.

Tout se passe donc comme si seule la prise en compte de l'altérité engageait la dimension problématique du jugement, y compris d'ailleurs au niveau de la philosophie première. Le Soll architectonique - voudrait-on suggérer -, pierre de touche de la possibilité de la conscience pure en I804, signale et n'exprime rien d'autre que la partition originaire entre l'être fermé sur soi et la liberté, le premier valant comme cette altérité avec laquelle la conscience ou image de l'être négocie son activité parce qu'elle y est «appelée» par lui. Le Soll spéculatif, note Marco Ivaldo, renvoie déjà d'une certaine manière à la sollicitation d'autrui car, dans l'esprit de Fichte, «Soll veut dire exigence (Forderung), mieux même: sollicitation (Auf-forderung) à la connaissance et à la réalisation de la vérité ${ }^{61}{ }$. A contrario, aucun «si... alors" ne semble pouvoir relativiser la proposition de l'éthique, à l'inverse de la proposition du droit et de la proposition fondamentale. Du moins, il en est ainsi au niveau qui est le sien, à savoir celui de la science appliquée, cette dernière demeurant bien entendu dépendante du Soll spéculatif. La déduction du principe de la moralité ne peut avoir elle-même de sens qu'eu égard à la possibilité ultime que la liberté soit. Ainsi, «le devoir moral, en tant que condition de la conscience de soi, semble dans cette mesure luimême dérivé et conditionné dans le procès déductif par l'hypothèse originaire de la réflexion transcendantale ${ }^{62} »$. Disons alors qu'une fois posée la dépendance du devoir moral envers le Soll originaire, le premier ne souffre aucune contingence dans sa propre énonciation. S'il doit y avoir une conscience, alors il doit y avoir une conscience morale, mais dès lors que celle-ci est admise, la conscience morale elle-même n'énonce rien d'autre que: «il doit y avoir un monde moral parce qu'il doit y en avoir un». C'est là pour Fichte une manière de dire que l'obligation morale (et non simplement l'existence de la moralité), contrairement à la règle de droit, ne dépend pas de l'entrée en relation avec autrui - dont la science première a déjà prouvé le caractère nécessaire s'il doit y avoir une conscience - et ne tolère aucune hypothéticité.

59. GA, I, 5, 66 .

60. GA, I, 5,67 .

61. M. Ivaldo, "Die konstitutive Funktion des Sollens in der Wissenschaftslehre", in E. Fuchs, M. Ivaldo et G. Moretto (dir.), Der transzendental-philosophische Zugang zur Wirklichkeit. Beiträge aus der aktuellen Fichte-Forschung, Stuttgart-Bad Cannstatt, Frommann-Holzboog, 200I, p. II4-II 5 .

62. Q. Landenne, Le Perspectivisme transcendantal de Fichte, op. cit., p. I25. 
Il est acquis que la sollicitation d'autrui constitue l'activité de la conscience (Bewusstsein) en son autonomie, en sensibilisant sa volonté en général mais aussi en instituant dans le droit son pouvoir d'agir autonome comme agir compromis. Il revient toutefois à la conscience morale (Gewissen) de faire de l'altérité, non plus la condition de la loi morale comme telle - car cette dernière est absolument ce qu'elle est par elle-même -, mais le partenaire indéfectible de son application. On retrouve ainsi l'intersubjectivité dans la troisième et dernière partie du Système, consacrée à l'application systématique du principe de la moralité. Examinant l'élément matériel de la loi morale, la seconde section de cette partie revient sur l'intercorporéité et la conscience de l'individualité du corps propre, en renvoyant explicitement au Droit naturel pour le détail. La sollicitation d'autrui réapparaît alors au titre de condition matérielle de l'autonomie, donc condition de possibilité d'une causalité effective, sensible, de la liberté, cette condition dépendant toutefois entièrement de la libre autoposition d'un moi devant catégoriquement faire être un monde moral ${ }^{63}$ :

mon égoïté et mon autonomie en général sont conditionnées par la liberté de l'autre; par conséquent, ma pulsion à l'autonomie ne peut absolument pas viser à nier la condition de sa propre possibilité, c'est-à-dire la liberté de l'autre ${ }^{64}$.

Non seulement la pulsion morale ne peut anéantir la pulsion morale d'autrui mais, tout au contraire, elle doit exiger moralement l'émancipation de tous. En effet, il importe qu'au plan matériel «le monde devienne pour moi ce qu'est pour moi mon corps ${ }^{65}$ ", c'est-à-dire l'instrument même de la liberté, l'un devant être en totale harmonie avec l'autre. Or, dans la mesure où les êtres raisonnables, par définition assujettis à la sollicitation, aspirent à vivre le monde comme leur propre corps, il faut admettre que «la manière de procéder conformément à la fin, chez l'un, est en même temps conforme à la fin pour tous les autres, que la libération de l'un est en même temps la libération de tous les autres ${ }^{66} »$. L'apparence individualiste de l'éthique fichtéenne se supprime: en s'efforçant d'accorder la volonté à elle-même, donc d'agir conformément à la loi morale et de transformer le monde en monde pour la liberté, le moi favorise d'abord nécessairement la liberté de l'autre. La seule manière moralement recevable d'accomplir son devoir est, pour

63. «Dans le Fondement du Droit naturel, la sollicitation est donc déduite du libre agir causal (Wirksamkeit) d'un moi individuel, mais dans le Système de l'éthique, elle l'est de l'autodétermination et spontanéité du moi originaire. Cette dernière déduction est le principe "supérieur" ". J.-Ch. Merle, «Die materiellen Bedingungen der Moralität (\$SI7-I8)», in J.-Ch. Merle et A. Schmidt (dir.), Fichtes System der Sittenlehre. Ein kooperativer Kommentar, Frankfurtam-Main, Vittorio Klostermann, 2015, p. I43.

64. GA, I, 5, $20 \mathrm{I}$.

65. GA, I, 5, 208.

66. GA, I, 5, 209. 
ainsi dire, que l'autre accomplisse le sien grâce à moi: « Ma fin est atteinte si l'autre agit moralement ${ }^{67}$.» Le moi et son autre ont bien sûr toujours la possibilité d'agir en opposition à la loi morale, précisément parce qu'ils sont libres, mais ils n'ont pas moins le devoir de se convaincre mutuellement d'agir moralement et par suite de redéfinir ensemble les normes qui incarnent la liberté ainsi mise en partage. Il s'agit d'une «action réciproque de tous avec tous en vue de la production (Hervorbringung) de convictions pratiques communes ${ }^{68} »$.

Sans la sollicitation d'autrui à réaliser son devoir dans le monde sensible, le moi serait incapable d'exprimer son autonomie, c'est-à-dire la loi de l'obligation morale au fondement de sa conscience. Considérée en ellemême, une telle loi peut néanmoins se dire de manière strictement catégorique, indépendamment de la survenue d'autrui, qui orientait en revanche immédiatement la nécessité juridique d'une limitation des sphères d'activité. D'un côté, le moi a le devoir d'œuvrer pour la liberté avec et pour autrui précisément parce que la loi de l'autonomie est catégoriquement ce qu'elle est indépendamment de l'autre, tandis qu'il a le droit, d'un autre côté, d'exiger la limitation réciproque des libertés si une sollicitation a lieu, événement qui seul rend toutefois possible la liberté elle-même. 